\title{
ON THE VANISHING CYCLES OF A MEROMORPHIC FUNCTION ON THE COMPLEMENT OF ITS POLES
}

\author{
DIRK SIERSMA AND MIHAI TIBĂR
}

\begin{abstract}
We study vanishing cycles naturally attached to a meromorphic function with isolated singularities, in both local and global settings.
\end{abstract}

\section{INTRODUCTION}

In the local setting, we consider a meromorphic function germ $p_{x} / q_{x}:(\mathcal{Z}, x) \rightarrow$ $\mathbb{P}^{1}(\mathbb{C})$, where $(\mathcal{Z}, x)$ is the germ of a complex manifold and $p_{x}$ and $q_{x}$ are holomorphic germs at the point $x$. By definition, $p_{x}^{\prime} / q_{x}^{\prime}$ is equal to $p_{x} / q_{x}$ if and only if there exists a holomorphic germ $u$ such that $u(x) \neq 0$ and that $p_{x}=u p_{x}^{\prime}$ and $q_{x}=u q_{x}^{\prime}$.

In the global setting, a meromorphic function $p / q: \mathcal{Z} \rightarrow \mathbb{P}^{1}(\mathbb{C})$ is defined as the ratio of two sections, $p$ and $q$, of a holomorphic line bundle $L \rightarrow \mathcal{Z}$ over a connected compact complex manifold $\mathcal{Z}$. We consider only examples of projective $\mathcal{Z}$, which case insures the existence of global meromorphic functions, by Kodaira embedding theorem.

Our constructions and results in the two settings are completely similar and parallel. This is why we shall adopt in this paper a unique notation for both situations: $p / q: \mathcal{Z} \rightarrow \mathbb{P}^{1}(\mathbb{C})$ can alternatively mean a meromorphic germ or a global meromorphic function.

The meromorphic function induces a holomorphic function $f: X \rightarrow \mathbb{C}$, on the complement of $X:=\mathcal{Z} \backslash\{q=0\}$ of the pole locus $\{q=0\}$. Then we call vanishing homology the relative homology $H_{*}(X, F ; \mathbb{Z})$, where $F$ denotes a general fiber of $f$ : $X \rightarrow \mathbb{C}$. Since $f$ is a non-proper function, vanishing cycles may appear not only because of the critical points of $f$, but also because of a certain non-regular behavior of the fibers of $f$ in the neighbourhood of the pole locus $\{q=0\}$, which is more subtle to detect. We interpret the later phenomenon in terms of singularities of the meromorphic function, to which we give a precise meaning. Our study concerns the class of meromorphic functions with isolated singularities, including possible singularities occuring in the indeterminacy locus. This is a class of non-generic pencils, far beyond the class of generic pencils that is currently considered in Lefschetz theory. We send the reader to [Ti4] and [Ti3] for comments on the relations to Lefschetz theory.

1991 Mathematics Subject Classification. Primary 32S50; Secondary 32A20, 32S30.

Key words and phrases. vanishing cycles, singularities along the indeterminacy locus, topology of pencils.

The authors benefited from the "Research in Pairs" program at Oberwolfach, supported by Volkswagen Foundation, and from the CNRS-NWO collaboration in research program, with support from the French side for the second author. 
While the homology of $X$ and that of $F$ might be very complicated, it turns out (Theorem 2.8) that the vanishing homology $H_{*}(X, F ; \mathbb{Z})$ is concentrated in dimension $n=\operatorname{dim} X$ and moreover that the space $X / F$ has the homotopy type of a bouquet of spheres $\bigvee S^{n}$. The proof needs new technical ingredients, due to the general notion of singularity that we use here. Proposition 3.2 and Lemma 3.3 are crucial in this respect.

We prove that the polar Milnor number which we attach to an isolated $\mathcal{G}$-singularity is the number of vanishing cycles which are "concentrated" at this singularity (Proposition 2.7 and Corollary 3.7). Alternatively, the polar Milnor number $\lambda_{\xi}$ at some point $\xi$ coincides with the jump of the usual local Milnor number of the pencil of hypersurfaces. Vanishing cycles can now be detected by a multibranch jump formula (Theorem 4.1).

We also show that the global vanishing homology decomposes into a direct sum of vanishing homologies at the atypical fibres, with localization in case of $\mathcal{G}$-isolated singularities (Proposition 2.7 and Corollary 3.7). We prove a general global Picard type formula for the monodromy (Proposition 5.1). Significant examples and particular cases are treated in 84 .

The vanishing homology in the context of meromorphic functions extends the one of local proper holomorphic functions, which has been initiated by Milnor [Mi] and developed in many aspects ever since. The meromorphic setting also generalizes the study of the topology of polynomial functions via their singularities at infinity and the study of one-parameter families of non compact hypersurfaces, developped in the last decade. (We send the reader to [Ti3] for more details on these connections.) New phenomena may occur: unlike the polynomial case, where singularities at infinity "stay at the same place" for all fibers, in the meromorphic case singularities can split or even disappear (see Example 4.4).

The results addressed here are based on our 1999 preprint [ST2], which has not been published. Ever since, there appeared several papers which quote it, like [DN2], GLM2], [Ti3]. The recent survey [Ti3] treats aspects of the topology of meromorphic functions on singular spaces, reviewing some of the results presented in the preprint [ST2] and in this paper. Connectivity results of Lefschetz type via nongeneric pencils (i.e. global meromorphic functions) are proved in [Ti4]. From a quite different point of view, meromorphic germs are discussed in [GLM1,2], where the main interest is the zeta-function of the monodromy. Classifications aspects have been explored by Arnold $[\mathrm{Ar}$, who found the list of simple germs of meromorphic functions under natural equivalence relations.

\section{Singularities of $f$ AlOng the indeterminacy LOCUS}

The definitions in this section naturally extend the ones used in case of polynomials and certain classes of regular functions on affine varieties, see [Ti1, Ti3] and also [Ti4]. According to our convention in the Introduction, we treat in parallel the local and global settings, using a common notation. 
Definition 2.1. We call completed space the global hypersurface $\mathbb{X}$ of $\mathcal{Z} \times \mathbb{P}^{1}$ defined by:

$$
s p(z)-t q(z)=0,
$$

where $[t: s] \in \mathbb{P}^{1}$. Then $\mathbb{X}$ is the analytic closure in $\mathcal{Z} \times \mathbb{P}^{1}$ of the graph of $f: X \rightarrow \mathbb{C}$. We denote by $\pi: \mathbb{X} \rightarrow \mathbb{P}^{1}$ the projection on $\mathbb{P}^{1}$. This is a proper holomorphic function which extends $f: X \rightarrow \mathbb{C}$. The space $\mathbb{X}^{\mathrm{pol}}:=\mathbb{X} \cap\left(\{p=q=0\} \times \mathbb{P}^{1}\right)$ is a divisor of $\mathbb{X}$ and $X$ is identified with $\mathbb{X} \backslash\left(\mathbb{X}^{\text {pol }} \cup \mathbb{X}_{\infty}\right)$, where $\mathbb{X}_{\infty}:=\pi^{-1}(\{s=0\})$.

We endow $\mathbb{X}$ with a locally finite Whitney stratification $\mathcal{W}$ such that $X$ is a stratum. In case of a germ at $x \in \mathcal{Z}$ of a meromorphic function one considers the germ of such a Whitney stratification at the line $\{x\} \times \mathbb{P}^{1} \subset \mathbb{X}$. In both situations, the local finiteness of the strata implies, by using Thom-Mather Isotopy Lemma [T] à la Verdier [Ve, the following finiteness result.

Proposition 2.2. The stratified projection $\pi: \mathbb{X} \rightarrow \mathbb{P}^{1}$ with respect to $\mathcal{W}$ is locally topologically trivial over $\mathbb{P}^{1} \backslash \Lambda_{f}$, for some finite set $\Lambda_{f} \subset \mathbb{P}^{1}$. In particular, the restriction $\pi_{\mid X}=f$ is a locally trivial $\mathrm{C}^{\infty}$-fibration over $\mathbb{C} \backslash \Lambda_{f}$.

We shall call the set of atypical values, and denote it by $\Lambda_{f}$, the minimal set $\Lambda_{f}$ which satisfies Proposition 2.2. Notice that, if we take the meromorphic function $q / p$ instead of $p / q$, then we get the same space $\mathbb{X}$ (even if $X$ will be $Z \backslash\{p=0\}$ instead of $Z \backslash\{q=0\})$. In particular, $\Lambda_{\frac{1}{f}}$ can be identified with $\Lambda_{f}$ by the isomorphism $[t: s] \mapsto[s: t]$.

For any subset $A \subset \mathbb{P}^{1}$, we denote $\mathbb{X}_{A}:=\pi^{-1}(A), F_{A}:=f^{-1}(A)$ and the general fibre $F:=F_{t}=X \cap f^{-1}(t)$, for some $t \notin \Lambda_{f}$. Let $D$ be a small disc centered at $a \in \Lambda_{f}$, such that $D \cap \Lambda_{f}=\{a\}$.

A crucial problem in investigating the topology of the fibres of $f$ is how to detect and to control the change of topology. This is an open problem, in general, even for holomorphic germs or polynomial functions, but well understood in case the singularities are isolated.

Definition 2.3. Let $(\mathcal{Z}, x) \rightarrow \mathbb{P}^{1}$ be a germ of a meromorphic function. To every $a \in$ $\mathbb{P}^{1}$, one associates the germ $\pi:(\mathbb{X},(x, a)) \rightarrow \mathbb{P}^{1}$. By restriction to $X=\mathbb{X} \backslash\left(\mathbb{X}^{\text {pol }} \cup \mathbb{X}_{\infty}\right)$, this defines the germ $f_{\mid}:(X,(x, a)) \rightarrow \mathbb{C}$, which we denote by $f_{x, a}$.

For some fixed $x \in\{q=p=0\}$, one has a one-parameter family of germs $(\mathbb{X},(x, a))$, indexed over $a \in \mathbb{P}^{1} \backslash\{s=0\}$. Unlike the case of holomorphic germs, here the point $(x, a)$ is not in $X$ but in its closure. So the germ $f_{x, a}$ is uniquely determined by the determination of the point $a=[p(x): q(x)]$.

Fourtheron, take a Whitney stratification $\mathcal{W}$ of $\mathbb{X}$ which has $X$ as open stratum, remarking that Sing $\mathbb{X} \subset \mathbb{X}^{\text {pol }}$. For all small enough radii $\varepsilon$ of a ball $B_{\varepsilon}(x, a) \subset \mathcal{Z} \times \mathbb{P}^{1}$ centered at $(x, a)$, the sphere $S_{\varepsilon}=\partial \bar{B}_{\varepsilon}(x, a)$ intersects transversally all the finitely many strata in the neighbourhood of $(x, a)$. This defines a Milnor-Lê fibration (cf [Lê2]), i.e. a locally trivial fibration $\pi: \mathbb{X}_{D^{*}} \cap B_{\varepsilon}(x, a) \rightarrow D^{*}$ over a small enough 
punctured disk $D^{*} \subset \mathbb{P}^{1}$ centered at $a$, which restricts to a locally trivial fibration on the complement of $\mathbb{X}^{\text {pol }}$, namely:

$$
f_{\mid}: F_{D^{*}} \cap B_{\varepsilon}(x, a) \rightarrow D^{*} .
$$

This fibration will be called the Milnor-Lê fibration of the function germ $f_{x, a}$ at the point $(x, a) \in \mathbb{X}$. It depends on the point $(x, a)$. In particular, the radius $\varepsilon$ of the ball depends on the point $a 1]$ From Proposition 2.2 it follows that, since $\pi$ is stratifiedtransversal to $\mathbb{X}$ over $\mathbb{P}^{1} \backslash \Lambda_{f}$, the fibration $f_{\mid}: F_{D} \cap B_{\varepsilon}(x, a) \rightarrow D$ is a trivial fibration, for all but a finite number of values of $a \in \mathbb{P}^{1}$, where $x \in\{p=q=0\}$ is fixed.

We now endow $\mathbb{X}$ with a "partial Thom stratification", cf [Ti1. Suppose that $\mathbb{X}$ is endowed with a complex stratification $\mathcal{G}=\left\{\mathcal{G}_{\alpha}\right\}_{\alpha \in S}$ such that $X$ is a stratum and $\mathbb{X}^{\text {pol }}$ is a union of strata. If $\mathcal{G}_{\alpha} \cap \overline{\mathcal{G}_{\beta}} \neq \emptyset$ then, by definition, $\mathcal{G}_{\alpha} \subset \overline{\mathcal{G}_{\beta}}$ and in this case we write $\mathcal{G}_{\alpha}<\mathcal{G}_{\beta}$.

Let $(x, a) \in \mathbb{X}^{\text {pol }} \backslash \mathbb{X}_{\infty}$. We consider on $\mathbb{X}$ the Thom $\left(\mathrm{a}_{q}\right)$ regularity condition at $(x, a)$, see e.g. GWPL, ch. I] for the definition. In terms of the relative conormal (see [Te], [HMS] $)$, the condition $\left(\mathrm{a}_{q}\right)$ at $\xi:=(x, a)$ for the strata $\mathcal{G}_{\alpha}$ and $\mathcal{G}_{\beta}$ translates to the inclusion: $T_{\mathcal{G}_{\alpha}}^{*} \supset\left(T_{q \mid \mathcal{G}_{\beta}}^{*}\right)_{\xi}$. It is known that this condition is independent on $q$, up to multiplication by a unit, see e.g. [Ti1, Prop. 3.2]. We therefore may and shall refer to this as Thom regularity condition relative to $\mathbb{X}^{\mathrm{pol}}$, at $(x, a)$.

Definition 2.4. We say that $\mathcal{G}$ is a $\partial \tau$-stratification (partial Thom stratification) relative to $\mathbb{X}^{\text {pol }}$ if at any point $\xi \in \mathbb{X}$, any two strata $\mathcal{G}_{\alpha}<\mathcal{G}_{\beta}$ with $\xi \in \mathcal{G}_{\alpha} \subset \mathbb{X}^{\text {pol }}$ and $\mathcal{G}_{\beta} \subset \mathbb{X} \backslash \mathbb{X}^{\infty}$ satisfy the Thom regularity condition relative to $\mathbb{X}^{\text {pol }}$.

The Whitney stratification $\mathcal{W}$ of $\mathbb{X}$ considered in Proposition 2.2 is an example of $\partial \tau$-stratification relative to $\mathbb{X}^{\mathrm{pol}}$. This follows from [BMM, Théorème 4.2.1] or [Ti1, Theorem 3.9]. Nevertheless the $\partial \tau$-stratifications are less demanding than Whitney stratifications and than Thom stratifications.

Definition 2.5. We consider the singular locus of $\pi$ with respect to some $\partial \tau$-stratification relative to $\mathbb{X}^{\mathrm{pol}}$, denoted by $\mathcal{G}$ and we say that the following closed subset of $\mathbb{X} \backslash \mathbb{X}{ }_{\infty}$ :

$$
\operatorname{Sing}_{\mathcal{G}} f:=\left(\mathbb{X} \backslash \mathbb{X}_{\infty}\right) \cap \cup_{\mathcal{G}_{\alpha} \in \mathcal{G}} \operatorname{closure}\left(\operatorname{Sing} \pi_{\mid \mathcal{G}_{\alpha}}\right)
$$

is "the singular locus of $f$ " with respect to $\mathcal{G}$. We say that $f$ has isolated singularities with respect to $\mathcal{G}$ if $\operatorname{dim}_{\operatorname{Sing}} f \leq 0$. We say that $f$ has isolated singularities at $a \in \mathbb{C}$ (or at the fibre $\mathbb{X}_{a}$ ) if $\operatorname{dim} \mathbb{X}_{a} \cap \operatorname{Sing}_{\mathcal{G}} f \leq 0$.

The space $X$ is nonsingular and consists of one stratum. The set $X \cap \operatorname{Sing}_{\mathcal{G}} f$ of $\mathcal{G}$ singularities on $X$ is just the usual singular set Sing $f \subset \mathcal{Z} \backslash\{q=0\}$. The singularities of the new type are $\mathbb{X}^{\text {pol }} \cap \operatorname{Sing}_{\mathcal{G}} f$.

We show that singularities of this type are manageable when they are isolated. In this case one may localize the variation of topology of fibres, which phenomenon has been observed before in the case of holomorphic germs, by Milnor [Mi], and in case

\footnotetext{
${ }^{1}$ One may compare to [GLM1,2], where different definitions have been used.
} 
of polynomial and regular functions [ST1, [Ti1]. Actually the proof for meromorphic functions follows the arguments of [Ti1, Theorem 4.3] and we leave it to the reader.

For instance, when $\operatorname{dim} \mathcal{Z}=2$, the pencil $p / q$ has isolated $\mathcal{G}$-singularities, relative to the coarsest partial Thom stratification $\mathcal{G}$, if and only if the fibres of $f$ are reduced.

Proposition 2.6. Let $f$ have isolated singularities with respect to $\mathcal{G}$ at $a \in \mathbb{C}$ and let $\mathbb{X}_{a} \cap \operatorname{Sing}_{\mathcal{G}} f=\left\{a_{1}, \ldots, a_{k}\right\}$. Then the variation of topology of the fibres of $f$ at $F_{a}$ is localizable at the points $a_{i}$.

The localization result implies that the vanishing cycles are concentrated at the isolated singularities, as follows:

Proposition 2.7. Let $f$ have isolated singularities with respect to $\mathcal{G}$ at $a \in \mathbb{C}$ and let $\mathbb{X}_{a} \cap \operatorname{Sing}_{\mathcal{G}} f=\left\{a_{1}, \ldots, a_{k}\right\}$. Let $D \subset \mathbb{C}$ be a small enough closed disc centered at a and let $s \in \partial D$. Then, for any small enough balls $B_{i} \subset \mathcal{Z} \times \mathbb{C}$ centered at $a_{i}$, we have:

(a) $H_{*}\left(F_{D}, F_{s}\right) \simeq \oplus_{i=1}^{k} \tilde{H}^{2 n-1-*}\left(B_{i} \cap \mathbb{X}_{s}\right)$.

(b) $H_{*}\left(B_{i} \cap F_{D}, B_{i} \cap F_{s}\right) \simeq \tilde{H}^{2 n-1-*}\left(B_{i} \cap \mathbb{X}_{s}\right), \forall i \in\{1, \ldots, k\}$.

Proof. Note first that in the local setting we have just one singular point, i.e. $k=1$. (a). A general Lefschetz duality result (see e.g. [Br, Prop. 5.2]) says that, since we work with triangulable spaces, we have:

$$
H_{*}\left(F_{D}, F_{s}\right) \simeq H^{2 n-*}\left(\mathbb{X}_{D}, \mathbb{X}_{s}\right) .
$$

Next, the cohomology group splits, through excision, into local contributions, by Proposition 2.6.

$$
H^{*}\left(\mathbb{X}_{D}, \mathbb{X}_{s}\right)=\oplus_{i=1}^{k} H^{*}\left(B_{i} \cap \mathbb{X}_{D}, B_{i} \cap \mathbb{X}_{s}\right)=\oplus_{i=1}^{k} \tilde{H}^{*-1}\left(B_{i} \cap \mathbb{X}_{s}\right),
$$

where the second equality holds because $B_{i} \cap \mathbb{X}_{s}$ is contractible, for small enough ball $B_{i}$.

(b). The same Lefschetz duality result may be applied locally to yield:

$$
H_{*}\left(B_{i} \cap F_{D}, B_{i} \cap F_{s}\right) \simeq H^{2 n-*}\left(B_{i} \cap \mathbb{X}_{D}, B_{i} \cap \mathbb{X}_{s}\right) .
$$

Note that the decomposition $H_{*}\left(F_{D}, F_{s}\right) \simeq \bigoplus_{i=1}^{k} H_{*}\left(B_{i} \cap F_{D}, B_{i} \cap F_{s}\right)$ also follows from 2.6 .

Our main result concerning vanishing cycles at the level of homotopy type is the following.

Theorem 2.8. Let $f$ have isolated singularities with respect to some $\partial \tau$-stratification $\mathcal{G}$ relative to $\mathbb{X}^{\mathrm{pol}}$. Let $F$ be a general fibre of $f$ and let $D \subset \mathbb{C}$ be a small enough open disc centered at some $a \in \Lambda_{f}$. Then the space $X$, resp. $F_{D}$, is obtained from $F$ to which one attaches a number of cells of real dimension $n=\operatorname{dim} \mathcal{Z}$. In particular we have the following homotopy equivalences:

(a) $X / F \simeq \bigvee S^{n}$, in the global setting.

(b) $F_{D} / F \simeq \bigvee S^{n}$, in the local or global setting. 
In the local setting, by $F_{D}$ and $F$ we mean the intersections with some small sphere $B_{\varepsilon}$, so (b) should read: $B_{\varepsilon} \cap F_{D} / B_{\varepsilon} \cap F \simeq \bigvee S^{n}$.

We shall give the proof in $\$ 3$, after introducing a few technical ingredients. The number of spheres will be discussed in Corollary 3.7. In the local setting, Theorem 2.8 extends Milnor's bouquet theorem [Mi], whereas in the global setting, it extends the bouquet result for polynomial functions [ST1, Theorem 3.1] and is of similar flavor as [Ti1, Theorem 4.6].

\section{Polar Curves and Milnor numbers at the indeterminacy locus}

We show first that an isolated $\mathcal{G}$-singularity at a point of $\mathbb{X}^{\text {pol }}$ is detectable by the presence of a certain local polar locus, which we define in the following.

Definition 3.1. Let $\xi=(x, a) \in \mathbb{X}^{\text {pol }} \backslash \mathbb{X}_{\infty}$ and consider a small neighbourhood $V \subset \mathcal{Z}$ of $x$. Let $\operatorname{Sing} f$, respectively $\operatorname{Sing}(f, q)$, denote the singular locus of the restriction $f: X \cap(V \times \mathbb{C}) \rightarrow \mathbb{C}$, respectively $(f, q): X \cap(V \times \mathbb{C}) \rightarrow \mathbb{C}^{2}$.

The polar locus $\Gamma_{\xi}(f, q)$ is the germ at $\xi$ of the analytic space:

$$
\text { closure }\left\{\operatorname{Sing}(f, q) \backslash\left(\operatorname{Sing} f \cup \mathbb{X}^{\text {pol }}\right)\right\} \subset \mathbb{X} \text {. }
$$

From the definition we get the isomorphism $\Gamma_{\xi}(f, q) \simeq \Gamma_{\xi}(p, q)$. The polar locus depends on the multiplicative unit $u$, i.e. $\Gamma_{\xi}(f, q u)$ is different from $\Gamma_{\xi}(f, q)$, meanwhile we shall prove that it induces well defined local invariants.

Proposition 3.2. Let $\xi=(x, a) \in \mathbb{X}^{\mathrm{pol}} \backslash \mathbb{X}_{\infty}$. Let $f$ have an isolated $\mathcal{G}$-singularity at $\xi$. Then:

(a) The polar locus $\Gamma_{\xi}(f, q u)$ is either void or $\operatorname{dim} \Gamma_{\xi}(f, q u)=1$, and this does not depend on the multiplicative unit $u$.

(b) The intersection multiplicity $\operatorname{mult}_{\xi}\left(\Gamma_{\xi}(f, q u), \mathbb{X}_{a}\right)$ is independent on the unit $u$.

Proof. For (a). we only give the rough idea and send for details to [ST2, Prop. 4.2] and [Ti3, Prop. 3.4]. The first claim follows by usual arguments, as in [Ti1]. The independence on $u$ is a consequence of the independence of the relative conormal $\mathbb{P T}_{q u}^{*}$ proved in [Ti1, Prop. 3.2].

If the polar locus is void, the multiplicity in (b) is zero. Suppose next that $\Gamma_{\xi}(f, q)$ has dimension 1. Consider a small enough ball $B \subset \mathcal{Z} \times \mathbb{C}$ centered at $\xi$, to fit in the Milnor-Lê fibration of the function $\pi$ at $\xi$ :

$$
\pi_{\mid}: B \cap \mathbb{X}_{D^{*}} \rightarrow D^{*}
$$

where $D \subset \mathbb{C}$ is centered at $a$. The notation $\Gamma(f, q)$ will stay for the representative in $B$ of the germ $\Gamma_{\xi}(f, q)$. We may choose $D$ so small, that for all $s \in \partial D$, those intersection points $\mathbb{X}_{s} \cap \Gamma(f, q)$ which tend to $\xi$ when $s$ tends to $a$, are inside $B$. This is possible because $\Gamma(f, q)$ is a curve which cuts $\mathbb{X}^{\text {pol }}$ at $\xi$.

We shall compute the homology $H_{*}\left(B \cap \mathbb{X}_{s}\right)$ of the Milnor fibre of the fibration (3.1). Inside $B$, the restriction of the function $q$ to $B \cap \mathbb{X}_{s}$ has a finite number of isolated singularities, which are precisely the points of intersection $B \cap \mathbb{X}_{s} \cap \Gamma(f, q)$. 
We claim that the space $B \cap \mathbb{X}_{s} \cap q^{-1}(\hat{\delta})$ is contractible, for small enough disc $\hat{\delta} \subset \mathbb{C}$ centered at 0 . To prove it, we need the following:

Lemma 3.3. Let $f$ have isolated $\mathcal{G}$-singularities at $\xi$. Let $B$ be a small enough ball at $\xi$ such that the sphere $S:=\partial \bar{B}$ cuts transversely all those finitely many strata of $\mathcal{G}$ which have $\xi$ in their closure and does not intersect other strata.

Then, there exist small enough discs $D$ and $\delta$ such that $(\pi, q)^{-1}(\nu)$ is transverse to $S$, for all $\nu \in D \times \delta^{*}$.

Proof. If the statement was not true, then there would exist a sequence of points $\eta_{i} \in S \cap\left(\mathbb{X} \backslash \mathbb{X}^{\text {pol }}\right)$ tending to a point $\eta \in S \cap \mathbb{X}_{a} \cap \mathbb{X}^{\text {pol }}$, such that the intersection of tangent spaces $T_{\eta_{i}} f^{-1}\left(f\left(\eta_{i}\right)\right) \cap T_{\eta_{i}} q^{-1}\left(q\left(\eta_{i}\right)\right)$ is contained in $T_{\eta_{i}}(S \cap X)$. Assuming, without loss of generality, that the following limits exist, we get the inclusion:

$$
\lim T_{\eta_{i}} f^{-1}\left(f\left(\eta_{i}\right)\right) \cap \lim T_{\eta_{i}} q^{-1}\left(q\left(\eta_{i}\right)\right) \subset \lim T_{\eta_{i}}(S \cap X) .
$$

Let $\mathcal{G}_{\alpha} \subset \mathbb{X}^{\text {pol }}$ be the stratum containing $\eta$. Remark that $\operatorname{dim} \mathcal{G}_{\alpha} \geq 2$, since $\overline{\mathcal{G}_{\alpha}} \ni \xi$ and $\pi \pitchfork_{\eta} \mathcal{G}_{\alpha}$. This implies that $\operatorname{dim} \mathcal{G}_{\alpha} \cap \mathbb{X}_{a} \geq 1$.

We have, by the definition of the stratification $\mathcal{G}$, that $\lim T_{\eta_{i}} q^{-1}\left(q\left(\eta_{i}\right)\right) \supset T_{\eta} \mathcal{G}_{\alpha}$ and obviously $T_{\eta}\left(\mathcal{G}_{\alpha} \cap \mathbb{X}_{a}\right) \subset T_{\eta} \mathcal{G}_{\alpha}$. On the other hand, $\lim T_{\eta_{i}} f^{-1}\left(f\left(\eta_{i}\right)\right) \supset T_{\eta}\left(\mathcal{G}_{\alpha} \cap \mathbb{X}_{a}\right)$, since $\pi \pitchfork_{\eta} \mathcal{G}_{\alpha}$. In conclusion, the intersection in (3.2) contains $T_{\eta}\left(\mathcal{G}_{\alpha} \cap \mathbb{X}_{a}\right)$. But, since $S \pitchfork_{\eta} \mathcal{G}_{\alpha}$, the limit $\lim T_{\eta_{i}}(S \cap X)$ cannot contain $T_{\eta}\left(\mathcal{G}_{\alpha} \cap \mathbb{X}_{a}\right)$ and this gives a contradiction.

Let $\hat{\delta}$ be so small that $B \cap \mathbb{X}_{s} \cap q^{-1}(\hat{\delta}) \cap \Gamma(f, q)=\emptyset$. By the Lemma 3.3 above and by choosing appropriate $D$ and $\hat{\delta}$, the map $q: B \cap \mathbb{X}_{s} \cap q^{-1}\left(\hat{\delta}^{*}\right) \rightarrow \hat{\delta}^{*}$ is a locally trivial fibration. Therefore $B \cap \mathbb{X}_{s} \cap q^{-1}(\hat{\delta})$ is homotopy equivalent, by retraction, to the central fibre $B \cap \mathbb{X}_{s} \cap \mathbb{X}^{\text {pol }}$. This proves our claim.

We now remark that the central fibre $B \cap \mathbb{X}_{s} \cap \mathbb{X}^{\text {pol }}$ is just the complex link at $\xi$ of the space $\mathbb{X}^{\text {pol }}$. The space $\mathbb{X}^{\text {pol }}$ is a product $(\{q=0\} \cap\{p=0\}) \times \mathbb{C}$ at $\xi$, along the projection axis $\mathbb{C}$, hence its complex link is contractible, and so is $B \cap \mathbb{X}_{s} \cap q^{-1}(\hat{\delta})$.

Pursuing the proof of Proposition 3.2, we observe that $B \cap \mathbb{X}_{s}$ is homotopy equivalent to $B \cap \mathbb{X}_{s} \cap q^{-1}(\delta)$, for $D$ and $\delta$ like in Lemma 3.3 and, in addition, the radius of $D$ much smaller than the radius of $\delta$. This supplementary condition is meant to insure that $\Gamma(f, q) \cap B \cap \mathbb{X}_{s}=\Gamma(f, q) \cap B \cap \mathbb{X}_{s} \cap q^{-1}(\delta)$.

Now, the total space $B \cap \mathbb{X}_{s} \cap q^{-1}(\delta)$ is built up by attaching to the space $B \cap$ $\mathbb{X}_{s} \cap q^{-1}(\hat{\delta})$, which is contractible, a finite number of cells of dimension $n-1$, which correspond to the Milnor numbers of the isolated singularities of the function $q$ on $B \cap \mathbb{X}_{s} \cap q^{-1}(\delta \backslash \hat{\delta})$. The sum of these numbers is, by definition, the intersection multiplicity $\operatorname{mult}_{\xi}\left(\Gamma(f, q), \mathbb{X}_{a}\right)$.

We have proven that:

$$
\operatorname{dim} H_{n-1}\left(B \cap \mathbb{X}_{s}\right)=\operatorname{mult}_{\xi}\left(\Gamma(f, q), \mathbb{X}_{a}\right) \text { and } \tilde{H}_{i}\left(B \cap \mathbb{X}_{s}\right)=0, \text { for } i \neq n-1
$$

When replacing all over in our proof the function $q$ by $q u$, we get the same relation (3.3), with $q u$ instead of $q$. This concludes our proof of 3.2 . 
The above proof shows that $B \cap \mathbb{X}_{s}$ is, homotopically, a ball to which one attaches a certain number of $(n-1)$-cells. We therefore get:

Corollary 3.4. Let $f$ have an isolated $\mathcal{G}$-singularity at $\xi$. The fibre $B \cap \mathbb{X}_{s}$ of the local fibration (3.1) is homotopy equivalent to a bouquet of spheres $\bigvee S^{n-1}$.

Definition 3.5. We denote the number of spheres by $\lambda_{\xi}:=\operatorname{dim} H_{n-1}\left(B \cap \mathbb{X}_{s}\right)$ and call it the polar Milnor number at $\xi$. We say that $f$ has vanishing cycles at $\xi$ if $\lambda_{\xi}>0$.

In the global setting, then we denote by $\lambda_{a}$ the sum of the polar Milnor numbers at singularities on $\mathbb{X}_{a} \cap \mathbb{X}^{\mathrm{pol}}$ and also denote $\lambda=\sum_{a \in \Lambda_{f}} \lambda_{a}$.

3.1. Proof of Theorem 2.8. We take back the notations of Theorem 2.8. Since Sing $f \subset \mathbb{X} \backslash \mathbb{X}_{\infty}$ is a finite set of points, the variation of topology of the fibres of $f$ is localizable at those points (cf. Proposition 2.6 ). Let $\mathbb{X}_{a} \cap \operatorname{Sing}_{\mathcal{G}} f=\left\{a_{1}, \ldots, a_{k}\right\}$, with $k=1$ in the local setting.

For some point $a_{i} \in X \cap \operatorname{Sing}_{\mathcal{G}} f$, by Milnor's classical result for holomorphic functions with isolated singularity [Mi], it follows that the pair $\left(B_{\varepsilon, i} \cap F_{D_{a}}, B_{\varepsilon, i} \cap F_{s}\right)$ is $(n-1)$ connected, where $s \in D_{a}^{*}$.

In case $a_{i} \in \mathbb{X}^{\text {pol }} \cap \operatorname{Sing}_{\mathcal{G}} f$, we may invoke the following lemma, which is a version of a result by Hamm and Lê [HL, Corollary 4.2.2] for our partial Thom stratification:

Lemma 3.6. ([Ti1, Cor. 2.7]) The pair $\left(B_{\varepsilon, i} \cap F_{D_{a}}, B_{\varepsilon, i} \cap F_{s}\right)$ is $(n-1)$-connected, where $s \in D_{a}^{*}$.

We conclude that the space $X$ is built up starting from a fibre $F$, then moving it within a fibration with a finite number of isolated singularities. By the above connectivity result and by Switzer's result [Sw Proposition 6.13], at each singular point one has to attach a number of cells of dimensions $\geq n$. In fact the cells to be attached are of dimension precisely $n$, by the following reason. We may apply the duality Proposition 2.7(b) and invoke Corollary 3.4, which show that the relative homology $H_{*}\left(F_{D_{a}}, F_{s}\right)$ is concentrated in dimension $n$.

Then one can map a bouquet of $n$ spheres into $F_{D} / F_{s}$ such that this map is an isomorphism in homology. This implies, by Whitehead's theorem, that the map induces an isomorphism of homotopy groups. (Remark that $F_{D} / F_{s}$ is simply connected whenever $n \geq 2$ ). Since we work with analytic objects, therefore triangulable, the space $F_{D} / F_{s}$ is a $\mathrm{CW}$-complex. For $\mathrm{CW}$-complexes, weak homotopy equivalence coincides with homotopy equivalence.

Let us remark that the total number of $n$-cells is the sum of the local Milnor numbers, resp. the polar Milnor numbers. This ends the proof of our theorem.

As consequence, we get the relative Betti numbers (see Proposition 2.7). This may be compared to similar formulas in case of polynomial functions [ST1].

Corollary 3.7. Let $f$ have isolated $\mathcal{G}$-singularities at $a \in \mathbb{C}$ with respect to some $\partial \tau$ stratification $\mathcal{G}$. Then $H_{j}\left(F_{D}, F_{s}\right)=0$ for $j \neq n$ and:

$$
b_{n}\left(F_{D}, F_{s}\right)=(-1)^{n} \chi\left(F_{D}, F_{s}\right)=\mu_{a}+\lambda_{a},
$$


where $\mu_{a}$ is the sum of the Milnor numbers of the singularities of $F_{a}$ and $\lambda_{a}$ denotes the sum of the polar Milnor numbers at $\mathbb{X}_{a} \cap \mathbb{X}^{\mathrm{pol}}$.

In particular, if $f$ has isolated $\mathcal{G}$-singularities at all fibres, then:

$$
b_{n}(X, F)=(-1)^{n} \chi(X, F)=\mu+\lambda, \quad H_{j}(X, F)=0, \text { for } j \neq n,
$$

where $\mu$ is the total Milnor number of the singularities of $f$ on $\mathcal{Z} \backslash\{q=0\}$ and $\lambda$ is the total polar Milnor number at $\mathbb{X}^{\mathrm{pol}} \backslash \mathbb{X}_{\infty}$.

\section{VANishing CYClES IN SPECIAL CASES AND EXAMPLES}

The singular locus Sing $\mathbb{X} \subset \mathcal{Z} \times \mathbb{C}$ is contained in $\mathbb{X}^{\mathrm{pol}}$ and can be complicated. We have Sing $\mathbb{X} \backslash \mathbb{X}_{\infty}=\cup_{t \in \mathbb{C}}\left(\operatorname{Sing} \mathbb{X}_{t}\right) \cap \mathbb{X}^{\text {pol }}$. However, $\mathbb{X}^{\text {pol }} \backslash \operatorname{Sing} \mathbb{X}$ is a Whitney stratum and Sing $\mathbb{X}$ is a union of Whitney strata, in the canonical Whitney stratification $\mathcal{W}$ of $\mathbb{X}$ which has $X$ as a stratum.

We shall consider here a $\partial \tau$-stratification $\mathcal{G}$ which may be coarser than $\mathcal{W}$ (and which exists, by Definition 2.4 and the remark following it). Then Sing $f \in \mathbb{X}^{\text {pol }} \subset$ Sing $\mathbb{X}$. Indeed, this follows from the fact that the space $\mathbb{X}^{\text {pol }} \backslash \mathbb{X}_{\infty}$ is locally a product $\{q=p=0\} \times \mathbb{C}$ and the projection $\pi$ is transversal to it off Sing $\mathbb{X}$.

In particular, for $n=2, f$ has isolated $\mathcal{G}$-singularities at $a \in \mathbb{C}$ if and only if $F_{a}$ is reduced.

Let $\xi=(x, a) \in \mathbb{X}^{\mathrm{pol}} \backslash \mathbb{X}_{\infty}$. We assume in the following that $\operatorname{dim}_{\xi} \operatorname{Sing} \mathbb{X}_{a}=0$. This implies that $\operatorname{dim}_{\xi} \operatorname{Sing}_{\mathcal{G}} f \leq 0$ and that the germ $(\operatorname{Sing} \mathbb{X}, \xi)$ is either a curve or just the point $\xi$. If it is a curve, then it can have several branches and its intersection with $\mathbb{X}_{s}$ is, say, $\left\{\xi_{1}(s), \ldots, \xi_{k}(s)\right\}$, for any $s \in D^{*}$, where $D \subset \mathbb{C}$ is a small enough disc at $a$.

The germs $\left(\mathbb{X}_{s}, \xi_{i}(s)\right)$ are germs of hypersurfaces with isolated singularity. Let $\mu_{i}(s)$ denote the Milnor number of $\left(\mathbb{X}_{s}, \xi_{i}(s)\right)$. Then $\sum_{i=1}^{k} \mu_{i}(s) \leq \mu(a)$. Equality may hold only if $k=1$, by the well known non-splitting result of Lê D.T [Lê1]. In general, we have:

Theorem 4.1. Let $\operatorname{dim}_{\xi} \operatorname{Sing} \mathbb{X}_{a}=0$ and $\operatorname{dim}_{\xi} \operatorname{Sing} \mathbb{X}=1$. Then:

$$
\lambda_{\xi}=\mu(a)-\sum_{i=1}^{k} \mu_{i}(s) \text {. }
$$

Proof. The hypothesis implies that the germ of $\operatorname{Sing}_{\mathcal{G}} f$ at $\xi$ is the point $\xi$ or it is void. For any $s \in D$ small enough, the germ $\left(\mathbb{X}_{s}, \xi_{i}(s)\right)$ is locally defined by the function:

$$
h=p-t q:\left(\mathcal{Z} \times \mathbb{C}, \xi_{i}(s)\right) \rightarrow \mathbb{C} .
$$

We have that, locally at $\xi$, the singular locus $\operatorname{Sing} h$ is equal to Sing $\mathbb{X}$, in particular included into $\mathbb{X}^{\text {pol }}$. Consider the map $(h, t):\left(\mathcal{Z} \times \mathbb{C}, \xi_{i}(s)\right) \rightarrow \mathbb{C}^{2}$. Note that the polar locus $\Gamma_{\xi}(h, t)$ is a curve or it is void, since $\xi$ is an isolated $\mathcal{G}$-singularity. Following [Lê2], there is a fundamental system of privileged polydisc neighbourhoods of $\xi$ in $\mathcal{Z} \times \mathbb{C}$, of the form $\left(P_{\alpha} \times D_{\alpha}^{\prime}\right)$, where $D_{\alpha}^{\prime} \subset \mathbb{C}$ is a disc at $a$ and $P_{\alpha}$ is a polydisc at $x \in \mathcal{Z}$ such 
that the map

$$
(h, t):(\mathcal{Z} \times \mathbb{C}) \cap\left(P_{\alpha} \times D_{\alpha}^{\prime}\right) \cap(h, t)^{-1}\left(D_{\alpha} \times D_{\alpha}^{\prime}\right) \rightarrow D_{\alpha} \times D_{\alpha}^{\prime}
$$

is a locally trivial fibration over $\left(D_{\alpha}^{*} \times D_{\alpha}^{\prime}\right) \backslash \operatorname{Im}(\Gamma(h, t))$. We chose $D_{\alpha}$ and $D_{\alpha}^{\prime}$ such that $\operatorname{Im}(\Gamma(h, t)) \cap \partial\left(\overline{D_{\alpha}^{*} \times D_{\alpha}^{\prime}}\right)=\operatorname{Im}(\Gamma(h, t)) \cap\left(D_{\alpha}^{*} \times \partial\left(\overline{D_{\alpha}^{\prime}}\right)\right)$. Let $s \in \partial \overline{D_{\alpha}^{\prime}}$. Observe that $t^{-1}(s) \cap\left(P_{\alpha} \times D_{\alpha}^{\prime}\right)$ is contractible, since it is the Milnor fibre of the linear function $t$ on a smooth space. This is obtained, up to homotopy type, by attaching to $(h, t)^{-1}(0, s) \cap$ $\left(P_{\alpha} \times D_{\alpha}^{\prime}\right)$ a certain number $r$ of $n$-cells, equal to the sum of the Milnor numbers of the function $h_{\mid}: t^{-1}(s) \cap\left(P_{\alpha} \times D_{\alpha}^{\prime}\right) \rightarrow D_{\alpha}$. Since we have the homotopy equivalence $(h, t)^{-1}(0, s) \cap\left(P_{\alpha} \times D_{\alpha}^{\prime}\right) \simeq B \cap \mathbb{X}_{s}$, we get, by Corollary 3.4 and Definition 3.5, that $r=\lambda_{\xi}$.

Now $(h, t)^{-1}(\eta, s) \cap\left(P_{\alpha} \times D_{\alpha}^{\prime}\right)$ is homotopy equivalent to the Milnor fibre of the germ $\left(\mathbb{X}_{a}, \xi\right)$, which has Milnor number $\mu(a)$. The space $t^{-1}(s) \cap\left(P_{\alpha} \times D_{\alpha}^{\prime}\right)$ is obtained from $(h, t)^{-1}(\eta, s) \cap\left(P_{\alpha} \times D_{\alpha}^{\prime}\right)$ by attaching exactly $r$ cells of dimension $n$ (coming from the polar intersections) and of a number of $n$-cells coming from the intersections with Sing $h$. This number of cells is, by definition, $\sum_{i=1}^{k} \mu_{i}(s)$. We get the equality $\mu(a)=r+\sum_{i=1}^{k} \mu_{i}(s)$. Since $r=\lambda_{\xi}$, our proof is done.

REMARK 4.2. If in the hypothesis of Theorem 4.1 the dimension of Sing $\mathbb{X}$ is not 1 but 0 , then the result still holds, with the remark that in this case $\mu_{i}(s)=0, \forall i$ and $\forall s \in D^{*}$. Hence $\lambda_{\xi}=\mu(a)$.

We give in the remainder three examples, one on $\mathbb{P}^{2}(\mathbb{C})$ with no singularities in the complement of $\mathbb{X}^{\mathrm{pol}}$ and two on a nonsingular quadratic surface in $\mathbb{P}^{3}(\mathbb{C})$.

ExAMPLE 4.3. $E_{p, q}^{a, b}: \quad f=\frac{x\left(z^{a+b}+x^{a} y^{b}\right)}{y^{p} z^{q}}$, with $a+b+1=p+q$ and $a, b, p, q \geq 1$.

This defines a meromorphic function on $\mathbb{P}^{2}(\mathbb{C})$. For some $t \in \mathbb{C}$, the space $\mathbb{X}_{t}$ is given by:

$$
x\left(z^{a+b}+x^{a} y^{b}\right)=t y^{p} z^{q}
$$

We have $\mathbb{X}^{\text {pol }} \backslash \mathbb{X}_{\infty}=\{[1: 0: 0],[0: 1: 0],[0: 0: 1]\} \times \mathbb{C}$. According to Theorem 4.1, we look for jumps in the Milnor number within the family of germs (4.1):

(a) at $[1: 0: 0]$, chart $x=1$. No jumps, since uniform Brieskorn type $(b, a+b)$.

(b) at $[0: 1: 0]$, chart $y=1$. For $t \neq 0$, Brieskorn type $(a+1, q)$, with $\mu(t)=a(q-1)$. If $t=0$, then we have $x^{a+1}+x z^{a+b}=0$ with $\mu(0)=a^{2}+a b+b$ and the jump at $\xi=([0: 1: 0], 0)$ is $\lambda_{\xi}=a^{2}+a b+b-a(q-1)=b+a p$, by Theorem 4.1],

(c) at $[0: 0: 1]$, chart $z=1$. No jumps, since type $A_{0}$ for all $t$.

We get the total jump $\lambda=b+a p$. A straightforward computation shows that $\mu=0$.

The fibres of $f$ can be described as follows. If $t=0$, we have $c+1$ disjoint copies of $\mathbb{C}^{*}$, where $c=\operatorname{gcd}(a, b)$; hence $\chi\left(F_{0}\right)=0$. If $t \neq 0$, we compute $\chi(F)=-(b+a p)$, by a branched covering argument. The vanishing homology is concentrated in dimension 2. Taking $X=\mathbb{C}^{2} \backslash\{y=0\}$, we get the Betti number $b_{2}(X, F)=\chi(X, F)=\chi(X)-$ 
$\chi(F)=0+(b+a p)=b+a p$. It follows $b_{2}(X, F)=\lambda+\mu$, which agrees with Corollary 3.7 .

EXAMPLE 4.4. Let $\mathcal{Z} \subset \mathbb{P}^{3}$ be the nonsingular hypersurface given by $h=x^{2}+z^{2}+y w=$ 0 and consider the meromorphic function $y / x$. It has its axis $\{x=y=0\}$ tangent to $h=0$ at $[0: 0: 0: 1]$. By computations we get $\mu=0, \lambda=1\left(\right.$ jump $A_{0} \rightarrow A_{1}$, at $\left.t=0\right)$. The general fibre $F$ is contractible, the special fibre $F_{0}$ is $\mathbb{C} \sqcup \mathbb{C}$ and $X$ is homotopy equivalent to $S^{2}$. All the connected components of fibres are contractible, however the global vanishing homology is generated by a relative 2 -cycle. We may remark here that the jump $A_{0} \rightarrow A_{1}$ cannot occur in case of polynomial functions at infinity.

EXAMPLE 4.5. Consider the meromorphic function $f=\frac{x\left(z^{2}+x y\right)}{z^{3}}$ on the nonsingular hypersurface $\mathcal{Z} \subset \mathbb{P}^{3}$ given by $h=y w+x^{2}-z^{2}=0$. Then $\mathbb{X}^{\text {pol }} \backslash \mathbb{X}_{\infty}=[0: 0: 0$ : 1] $\times \mathbb{C} \cup[0: 1: 0: 0] \times \mathbb{C}$, where $[x: y: z: w]$ are the homogeneous coordinates in $\mathbb{P}^{3}$.

Along $[0: 0: 0: 1] \times \mathbb{C}$, in the chart $w=1$ and coordinates $x$ and $z$ on $\mathbb{X}$, we have the family of curves (germs of $\mathbb{X}_{t}$ ):

$$
x\left(z^{2}-x^{3}+x z^{2}\right)=t z^{3} .
$$

For all $t$, this is a $D_{5}$ singularity, so no jumps.

Along $[0: 1: 0: 0] \times \mathbb{C}$, in the chart $y=1$ and, again, $x$ and $z$ as coordinates on $\mathbb{X}$, we have the family of curves (germs of $\mathbb{X}_{t}$ ):

$$
x\left(z^{2}+x\right)=t z^{3} .
$$

This has type $A_{2}$ if $t \neq 0$ and $A_{3}$ if $t=0$. Thus the jump at $\xi:=([0: 1: 0: 0], 0)$ is $\lambda_{\xi}=1$ and the total jump is $\lambda=1$.

By simple computations, we get $\mu=2$, since there are two singular fibres, $F_{ \pm 1}$, with $A_{1}$-singularities. There are 3 atypical fibres: $F_{0} \simeq \mathbb{C}^{*} \sqcup \mathbb{C}^{*}, F_{ \pm 1} \simeq \mathbb{C}^{*}$ and the general fibre $F \simeq \mathbb{C}^{* *}$. Since $X \simeq S^{2}$, we get $b_{2}(X, F)=2-(-1)=3$ global vanishing cycles, $X / F \simeq \bigvee_{3} S^{2}$

\section{Monodromy fibration And a GLOBAl PiCARD PHENOMEnon}

We continue to consider the local and global settings in the same time. Let $\hat{D} \subset \mathbb{C}$ be a closed disc, big enough such that $\hat{D} \supset \Lambda_{f} \backslash \infty$, where $\infty$ denotes the point $[1: 0] \in \mathbb{P}^{1}$. Let $D_{i} \subset \hat{D}$ be a small enough closed disc at $a_{i} \in \Lambda_{f}$, such that $D_{i} \cap \Lambda_{f}=\left\{a_{i}\right\}$. Take a point $s$ on the boundary of $\hat{D}$ and, for each $i$, a path $\gamma_{i} \subset \hat{D}$ from $s$ to some fixed point $s_{i} \in \partial D_{i}$, with the usual conditions: the path $\gamma_{i}$ has no self intersections and does not intersect any other path $\gamma_{j}$, except at the point $s$. By Proposition 2.2, the fibration $f: X \backslash f^{-1}\left(\Lambda_{f}\right) \rightarrow \mathbb{C} \backslash \Lambda_{f}$ is locally trivial, hence we may use excision in the pair $\left(F_{\hat{D}}, F_{s}\right)$ and get an isomorphism (induced by the inclusion of pairs):

$$
\oplus_{a_{i} \in \Lambda_{f}} H_{*}\left(F_{D_{i}}, F_{s_{i}}\right) \rightarrow H_{*}\left(X, F_{s}\right),
$$


This shows that each inclusion $\left(F_{D_{i}}, F_{s_{i}}\right) \subset\left(X, F_{s_{i}}\right)$ induces an injection in homology $H_{*}\left(F_{D_{i}}, F_{s_{i}}\right) \hookrightarrow H_{*}\left(X, F_{s_{i}}\right)$. We also get by excision the following split exact sequence:

$$
0 \rightarrow H_{*}\left(F_{D_{i}}, F\right) \rightarrow H_{*}(X, F) \rightarrow \oplus_{a_{j} \in \Lambda_{f}, j \neq i} H_{*}\left(F_{D_{j}}, F\right) \rightarrow 0 .
$$

We next consider the monodromy $h_{i}$ around an atypical value $a_{i} \in \Lambda_{f}$. This is induced by a counterclockwise loop around the small circle $\partial D_{i}$. The monodromy acts on the pair $(X, F)$ and we denote its action in homology by $T_{i}$.

The following sequence of maps:

$$
H_{q+1}(X, F) \stackrel{\partial}{\rightarrow} H_{q}(F) \stackrel{w}{\rightarrow} H_{q+1}\left(F_{\partial D_{i}}, F\right) \stackrel{i_{*}}{\rightarrow} H_{q+1}(X, F),
$$

where $w$ denotes the Wang map (which is an isomorphism, by the Künneth formula), gives, by composition, the map $T_{i}-\mathrm{id}: H_{q+1}(X, F) \rightarrow H_{q+1}(X, F)$.

This overlaps the first two maps in the following sequence: $H_{q}(F) \stackrel{w}{\rightarrow} H_{q+1}\left(F_{\partial D_{i}}, F\right) \stackrel{i_{*}}{\rightarrow}$ $H_{q+1}(X, F) \stackrel{\partial}{\rightarrow} H_{q}(F)$. The last arrow in the sequence (5.2) fits in the commutative diagram:

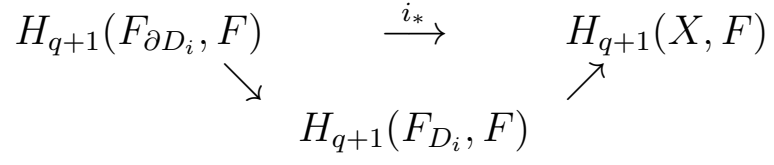

where all three arrows are induced by inclusion.

It follows that the submodule of "anti-invariant cycles" $\mathcal{I}_{*}\left(T_{i}\right):=\operatorname{Im}\left(T_{i}-\right.$ id : $\left.H_{*}(X, F) \rightarrow H_{*}(X, F)\right)$ is contained in the direct summand $H_{*}\left(F_{D_{i}}, F\right)$ of $H_{*}(X, F)$. If $\mathcal{I}_{*}$ denotes the submodule generated by $\mathcal{I}_{*}\left(T_{i}\right)$, for all $a_{i} \in \Lambda_{f}$, then:

$$
\mathcal{I}_{*}=\oplus_{a_{i} \in \Lambda_{f}} \mathcal{I}_{*}\left(T_{i}\right) \text {. }
$$

Using Picard's decomposition of the monodromy, Lefschetz has proven the famous relation for the monodromy around a simple nodal singularity on a nonsingular ambient space, wellknown as Picard-Lefschetz formula. The following result describes a global Picard phenomenon.

Proposition 5.1. Identify $H_{*}(X, F)$ to $\oplus_{a_{i} \in \Lambda_{f}} H_{*}\left(F_{D_{i}}, F\right)$ by the isomorphism (5.1). Then, for $\omega \in H_{*}(X, F)$, we have:

$$
T_{i}(\omega)=\omega+\psi_{i}(\omega)
$$

for some $\psi_{i}(\omega) \in H_{*}\left(F_{D_{i}}, F\right)$.

In the global setting, when specializing to a homologically contractible total space $X$, the natural $\partial$-map $H_{*}(X, F) \rightarrow \tilde{H}_{*-1}(F)$ becomes an isomorphism and we get: $\tilde{H}_{*}(F)=\oplus_{a_{i} \in \Lambda_{f}} H_{*+1}\left(F_{D_{i}}, F\right)$. This occurs for instance in case of a polynomial function $g: \mathbb{C}^{n} \rightarrow \mathbb{C}$, for which $X=\mathbb{C}^{n}$. Results on invariant cocycles were obtained in NN1. In our more general setting, these results can also be proved by dualizing from homology to cohomology. One obtains in this way statements about invariant cocycles $\operatorname{Ker}\left(T^{i}-\mathrm{id}: H^{*}(X, F) \rightarrow H^{*}(X, F)\right)$ instead of anti-invariant cycles. 
In the particular case of polynomial functions, the above Picard formula (extracted from our preprint [ST2]) was independently noticed in [NN2] and [DN1].

We end by an easy consequence, remarked in the special case of polynomial functions in [DN2, which holds in our more general setting of local and global meromorphic functions.

Corollary 5.2. Assume that the number of paths is $l$ and the paths $\gamma_{1}, \ldots \gamma_{l}$ are counterclockwise ordered. Then the Coxeter element $T_{\infty}:=T_{l} \circ \cdots \circ T_{1}$ determines the generators $T_{i}, \forall i \in\{1, \ldots, l\}$.

Proof. Use the direct sum decomposition (5.3) together with the following adapted decomposition of $T_{\infty}-\mathrm{id}$, where we couple two-by-two consecutive terms: $T_{\infty}-\mathrm{id}=$ $\left(T_{l} \circ \cdots \circ T_{1}-T_{l-1} \circ \cdots \circ T_{1}\right)+\ldots+\left(T_{1}-\mathrm{id}\right)$.

\section{REFERENCES}

[Ar] V.I. Arnold, Singularities of fractions and behaviour of polynomials at infinity, Tr. Mat. Inst. Steklova 221 (1998), 48-68.

[BMM] J. Briançon, Ph. Maisonobe, M. Merle, Localisation de systèmes différentiels, stratifications de Whitney et condition de Thom, Inventiones Math. 117, 3 (1994), 531-550.

[Br] S.A. Broughton, On the topology of polynomial hypersurfaces, Proceedings A.M.S. Symp. in Pure. Math., vol. 40, I (1983), 165-178.

[DN1] A. Dimca, A. Némethi, Thom Sebastiani construction and monodromy of polynomials, Université de Bordeaux, preprint no. 98/1999.

[DN2] A. Dimca, A. Némethi, On monodromy of complex polynomials, Duke Math. J. 108 (2001), no. 2, 199-209. (arXiv:math.AG/9912072).

[GWPL] C.G. Gibson, K. Wirthmüller, A.A. du Plessis, E.J.N. Looijenga, Topological Stability of Smooth Mappings, Lect. Notes in Math. 552, Springer Verlag 1976.

[GLM1] S. Gusein-Zade, I. Luengo, A. Melle, Zeta functions for germs of meromorphic functions and Newton diagrams, Funct. Anal. Appl. 32, 2 (1998), 93-99.

[GLM2] S. Gusein-Zade, I. Luengo, A. Melle, Bifurcations and topology of meromorphic germs, in: New developments in singularity theory (Cambridge, 2000), 279-304, NATO Sci. Ser. II Math. Phys. Chem., 21, Kluwer Acad. Publ., Dordrecht, 2001.

[HL] H. Hamm, Lê D.T., Rectified homotopical depth and Grothendieck conjectures, in: P. Cartier et all. (eds) Grothendieck Festschrift II, pp. 311-351, Birkhäuser 1991.

[HMS] J.P. Henry, M. Merle, C. Sabbah, Sur la condition de Thom stricte pour un morphisme analytique complexe, Ann. Scient. Ec. Norm. Sup. $4^{e}$ série, t. 17 (1984), 227-268.

[Lê1] Lê D.T., Une application d'un théorème d'A'Campo à l'équisingularité, Nederl. Akad. Wet., Proc., Ser. A 76, 403-409 (1973).

[Lê2] Lê D.T., Some remarks on the relative monodromy, in: Real and Complex Singularities, Oslo 1976, Sijhoff en Norhoff, Alphen a.d. Rijn 1977, p. 397-403.

[Mi] J. Milnor, Singular points of complex hypersurfaces, Ann. of Math. Studies 61, Princeton 1968.

[NN1] W. D. Neumann, P. Norbury, Vanishing cycles and monodromy of complex polynomials, Duke Math. J. 101, no. 3(2000), 487-497.

[NN2] W. D. Neumann, P. Norbury, Unfolding polynomial maps at infinity, Math. Ann. 318, no. 1 (2000), 149-180.

[ST1] D. Siersma, M. Tibăr, Singularities at infinity and their vanishing cycles, Duke Math. Journal 80:3 (1995), 771-783. 
[ST2] D. Siersma, M. Tibăr, Vanishing cycles and singularities of meromorphic functions, Utrecht University preprint 1105, May 1999. arXiv:math.AG/9905108.

[Sw] R. Switzer, Algebraic Topology - Homotopy and Homology, Springer Verlag, BerlinHeidelberg-New York 1975.

[Te] B. Teissier, Varietés polaires 2: Multiplicités polaires, sections planes et conditions de Whitney, Géométrie Algèbrique à la Rabida, Springer L.N.M. 961, pp. 314-491.

[T] R. Thom, Ensembles et morphismes stratifiés, Bull. Amer. Math. Soc. 75 (1969), 249-312.

[Ti1] M. Tibăr, Topology at infinity of polynomial maps and Thom regularity condition, Compositio Math., 111, 1 (1998), 89-109.

[Ti2] M. Tibăr, Asymptotic Equisingularity and Topology of Complex Hypersurfaces, Int. Math. Research Notices 1998, no. 18, 979-990.

[Ti3] M. Tibăr, Singularities and Topology of Meromorphic Functions, in: A. Libgober, M. Tibăr (eds.) Trends in Singularities, pp. 223-246, Birkhäuser 2002.

[Ti4] M. Tibăr, Connectivity via nongeneric pencils, Internat.J.Math., 13, 2 (2002), 111-123.

[Ve] J.-L. Verdier, Stratifications de Whitney et théorème de Bertini-Sard, Inventiones Math. 36 (1976), 295-312.

D.S.: Mathematisch Instituut, Universiteit Utrecht, PO Box 80010, 3508 TA Utrecht, The NetherLANDS.

E-mail address: siersma@math.uu.nl

M.T.: Mathématiques, UMR 8524 CNRS, Université de Lille 1, 59655 Villeneuve D'ASCQ, France.

E-mail address: tibar@agat.univ-lille1.fr 